Pacific Journal of Mathematics

NILPOTENT OR A RIGHT MULTIPLE IS A SYMMETRIC
IDEMPOTENT

G. P. WEN 


\title{
ALTERNATIVE RINGS WHOSE SYMMETRIC ELEMENTS ARE NILPOTENT OR A RIGHT MULTIPLE IS A SYMMETRIC IDEMPOTENT
}

\author{
G. P. WenE
}

\begin{abstract}
Osborn characterizes those associative rings with involutions in which each symmetric element is nilpotent or invertible. Analogous results are obtained for alternative rings. The restriction is further relaxed to require only that each symmetric element is nilpotent or some multiple is a symmetric idempotent.
\end{abstract}

Introduction. J. M. Osborn [10] [11] proved a series of theorems concerning the structure of associative rings with involution such that any symmetric element is either nilpotent or invertible. Many generalizations of his results have appeared in the literature for associative rings (a good single reference is Herstein [4]). We begin with a discussion of involutions in the Cayley-Dickson algebras. Then Osborn's results are generalized to alternative rings. Our final result shows that if $R$ is an alternative ring with involution * such that (a) each symmetric element $s$ is either nilpotent or some right multiple of $s$ is a symmetric idempotent and (b) each set of pairwise orthogonal symmetric idempotent has $n$ or less elements, then the quotient $\operatorname{ring} R / \operatorname{Rad} R$ has d.c.c on right ideals. Since a radical free alternative ring with d.c.c. on right ideals is the direct sum of Cayley-Dickson algebras and simple artinian associative rings, we have a nice description of these quotient rings.

1. Preliminaries. Let $R$ be a nonassociative ring. As is usual for $x, y, z$ in $R$ we denote the associator $(x y) z-x(y z)$ by $(x, y, z)$ and the commutator $x y-y x$ by $[x, y] . \quad R$ is alternative if $(x, x, y)=$ $(x, y, y)=0$ and is Jordan if $\left(x^{2}, y, x\right)=[x, y]=0$. The alternative and Jordan rings are flexible in the sense that $(x, y, x)=0$ for all $x, y$. If $R$ is an algebra over a field $\Phi$ not of characteristic two, and $A$ a subset of $R$, then $A^{+}$will denote the set of elements of $R$ generated by the elements of $A$ under the addition of $R$ and Jordan product $a \circ b=1 / 2(a b+b a)$ where $a b$ denotes the product as elements of $R . A^{-}$is defined similarly except that the product is now $[a, b]=$ $a b-b a$.

The radical, $\operatorname{Rad} R$, of an alternative ring is the maximal ideal consisting entirely of elements $z$ which are quasi-invertible in the sense that $1-z$ is invertible. A ring is called a radical ring if $R=\operatorname{Rad} R . \quad$ If $\operatorname{Rad} R=0$, then $R$ is said to be radical free. 
An ideal $I$ in a ring $R$ with involution $*$ is a $*$-ideal if $I^{*}=I$. $R$ is *-simple if the only *-ideals of $R$ are $R$ and 0 and $R$ is not trivial $\left(R^{2} \neq 0\right) . \quad R$ is *-simple if and only if $R$ is simple or $R$ is the direct sum of a simple ring and its opposite.

LEMMA 1. If $R$ is an alternative ring with involution and each symmetric element is nilpotent, then $R$ is a radical ring.

Proof. The proof that Osborn [11] uses in the associative case works here.

REMARK. The converse of the lemma is false. Golovena [2] constructs radical subrings of the ring of rational numbers. Since these rings are commutative, the identity map is an involution. But no nonzero element of the rational numbers is nilpotent.

We use the fact that Cayley-Dickson algebras are quadratic. An algebra $\mathscr{U}$ with identity $e$ over a field $\Phi$ not of characteristic 2 is called quadratic if $\mathscr{U} \neq \Phi e$ and for each $x$ in $\mathscr{U}$

$$
x^{2}-2 t(x) x+q(x) e=0
$$

where $t(x)$ and $q(x)$ are in $\Phi$ and $t(\alpha e)=\alpha$ for all $\alpha \in \Phi$. In what follows $\Phi$ has more than 2 elements. The quantities $t(x)$ and $q(x)$ in equation 1 are called the trace and norm of $x$, respectively. The trace is a linear functional on $\mathscr{U}$ (see Schafer [14], p. 49). The norm $q(x)$ defines a symmetric bilinear form $q(x, y)$, the norm form, on $\mathscr{U}$ via

$$
q(x, y)=q(x+y)-q(x)-q(y) .
$$

Say $q(x)$ is nondegenerate if $q(x, y)$ is nondegenerate.

A quadratic algebra $\mathscr{C}$ will be flexible if and only if the trace $t(x)$ is associative; that is, $t((x, y, z))=0$ for all $x, y, z$ in $\mathscr{U}$. $\mathscr{U}^{+}$ will always be a Jordan algebra; $\mathscr{C}^{+}$will be simple if and only if $t(x)$ is associative and $q(x)$ is nondegenerate (Braun and Koecher [1], p. 217). If $\mathscr{C}$ possesses a symmetric bilinear form $f(x, y)$ such that $f(x y, z)=f(x, y z)$ (an invariant form) and $f(x, y)$ is nondegenerate on $\mathscr{U}$, then $\mathscr{U}^{\circ}=\mathscr{U}^{-} \mid Z\left(\mathscr{U}^{-}\right), Z\left(\mathscr{U}^{-}\right)=\left\{x \in \mathscr{U}^{-} \mid[x, y]=0\right.$ for all $\left.y \in \mathscr{C}^{-}\right\}$, is simple (Sagle [13]). Any Cayley-Dickson algebra has a nondegenerate invariant form given by $f(x, y)=\operatorname{trace}\left(R_{x} R_{y}\right)$ (Schafer [14], p. 44).

Every flexible, quadratic algebra $\mathscr{C}$ has an involution $x \rightarrow \bar{x}$ where

$$
\bar{x}=2 t(x) e-x .
$$

Furthermore $x \rightarrow \bar{x}$ is the unique involution $*$ such that 


$$
(\alpha e)^{*}=\alpha e, x+x^{*} \in \Phi e \text { and } x x^{*}=x^{*} x \in \Phi e
$$

for all $\alpha \in \Phi$ and $x \in \mathscr{U}$. Clearly any algebra $\mathscr{U}$ with involution * satisfying (2) is quadratic. Call this involution satisfying (2) the standard involution.

REMARK. If $\mathscr{U}$ is quadratic and $x \rightarrow \bar{x}=2 t(x) e-x$ is an involution in $\mathscr{U}, \mathscr{C}$ does not need to be flexible (see Braun and Koecher [1], p. 217).

2. Involutions in Cayley-Dickson algebras. A simple alternative ring, which is not associative, is a Cayley-Dickson algebra over its center, $Z$ (Kleinfeld [8]). The involutions in simple artinian associative rings are determined in Jacobson and Rickart [7]. We now determine the involutions in Cayley-Dickson algebras.

Let $\mathscr{C}$ be a Cayley-Dickson algebra not of characteristic two with involution *. $S$ will denote the set of symmetric elements and $K$ will denote the set of skew elements. Let $Z$ denote the center of $\mathscr{C}$. As in the associative case, an involution $*$ is said to be of the first kind if $Z \cong S$; otherwise $*$ is of the second kind. $\mathscr{C}$ has a vector space decomposition over $Z \cap S, \mathscr{C}=S+K$.

Lemma 2. Let $\mathscr{C}$ have an involution * of the first kind. Then one of the following is true:

(a) * is the standard involution - in $\mathscr{C}, S=Z, S^{+}$is isomorphic to $Z$, and $K^{-}$is a simple 7-dimensional algebra over $Z$.

(b) $S^{+}$is a simple, 5-dimensional algebra over $Z$ and $K^{-}$is a simple, 3-dimensional Lie algebra over $Z$.

Proof. Let $*$ be an involution of the first kind. Then $f(x)=$ $\overline{x^{*}}$ is a $Z$-linear automorphism of $\mathscr{C}, \overline{f(x)}=x^{*}$, and $\overline{f(x)}=2 t(f(x)) e-$ $f(x)=f(2 t(x) e-x)=f(\bar{x})$, since automorphisms preserve the trace. Hence $x^{*}=\overline{f(x)}=f(\bar{x})$ and $\left.\left(x^{*}\right)^{*}=\overline{f(f(\bar{x})}\right)=f(f(x))=x$. That is, $f$ is an automorphism of period two.

If $f$ is the identity map, then $*$ is the standard involution -. The set $S$ of symmetric elements is the center $Z$ and $S^{+}=Z^{+}=Z$ is a field. Since $K^{-}$is isomorphic to $\mathscr{C}^{0}$, it is simple. We note for later use that $K=[\mathscr{C}, \mathscr{C}]=[[\mathscr{C}, \mathscr{C}],[\mathscr{C}, \mathscr{C}]]$.

As in Jacobson [5], we call an automorphism of period two a reflection if it is not the identity map. If $f$ is a reflection in $\mathscr{C}$ then there is a nonisotropic, quaternion subalgebra $\mathscr{B}$, where $\mathscr{C}=\mathscr{B}+\mathscr{B} h, h \in \mathscr{B}^{\perp}, q(h, h) \neq 0$ and $f\left(\beta_{0}+\beta_{1} h\right)=\beta_{0}-\beta_{1} h$ for each $\beta_{0}, \beta_{1}$ in $\mathscr{B}$. Since $x^{*}=\overline{f(x)}$, we have $\left(\beta_{0}+\beta_{1} h\right)^{*}=\beta_{0}-\beta_{1} h=$ $\bar{\beta}_{0}+\beta_{1} h$. That is $S=Z e+\mathscr{B} h$ and the dimension of $S$ over $Z$ is 
five. Since the norm form is nondegenerate on $S, S^{+}$is a simple Jordan algebra. $K^{-}$, being generated by a subset of the (necessarily associative) quaternion algebra $\mathscr{B}$, is Lie. Since the invariant form of $\mathscr{C}$ restricted to $\mathscr{B}$ is nondegenerate, $\mathscr{B}^{0}$ is simple and $K^{-}$, being isomorphic to $\mathscr{B}^{0}$, is simple.

LEMma 3. Let $*$ be an involution in $\mathscr{C}$ of the second kind. Then there is an element $\lambda, \lambda \neq 0, \lambda \in Z$ such that $\lambda^{*}=-\lambda, \mathscr{C}=$ $S+\lambda S$ and $S^{+}$and $K^{-}=(\lambda S)^{-}$are simple.

Proof. As in the associative case, there is $\lambda \neq 0, \lambda \in Z$ such that $\lambda^{*}=-\lambda, K=\lambda S$ and $\mathscr{C}=S+\lambda S$. Since $\tilde{\mathscr{C}}=\mathscr{\mathscr { C }} \otimes_{F} L$ is quadratic over $L$ if and only if $\mathscr{U}$ is quadratic over $F$ (Schafer [14], p. 50) and the fact that the norm form is nondegenerate on $\mathscr{C}^{+}=S^{+} \boldsymbol{\otimes}_{z_{0}} Z$, the norm form is nondegenerate on $S^{+}$. Hence $S^{+}$is simple.

Let $k=\lambda^{-1}$. Then $\mathscr{C}=K+k K$ and $[\mathscr{C}, \mathscr{C}]=[K, K]+k[K, K]+$ $k^{2}[K, K]$ and since $k^{2} \in Z_{0}, k^{2}[K, K]=[K, K]$. Thus $[K, K]+k[K, K]=$ $[\mathscr{C}, \mathscr{C}]=[[\mathscr{C}, \mathscr{C}],[\mathscr{C}, \mathscr{C}]]=[[K, K],[K, K]]+k[[K, K],[K, K]]$. Since $[[K, K],[K, K]]$ is contained in $[K, K]$, we have $[[K, K],[K, K]]=[K, K]$.

Let $I$ be an ideal of $[K, K]$. Then $I+Z I \subset[\mathscr{C}, \mathscr{C}]$ and $[I+Z I$, $[\mathscr{C}, \mathscr{C}]] \subset[I+Z I,[K, K]]+[I+Z I, k[K, K] \subset[I,[K, K]]+k[I,[K, K]+$ $Z[I,[K, K]+Z k[I,[K, K]] \subset Z I+I$. Thus $I+Z I$ is an ideal of $[\mathscr{C}, \mathscr{C}]$. But by the proof of Lemma $2,[\mathscr{C}, \mathscr{C}]$ is simple. If $I+Z I=(0)$, then $I=(0)$. On the other hand, suppose $I+Z I=[\mathscr{C}, \mathscr{C}]=[K, K]+$ $k[K, K]$. But $I+Z I=I+Z_{0} I+k Z_{0} I$. Hence $I+Z_{0} I=[K, K]$ and $I \supset[I$, $[K, K]]=\left[I, I+Z_{0} I\right] \supset[I, I]+Z_{0}[I, I]$, and $[K, K]=[[K, K],[K, K]]=$ $\left[I+Z_{0} I, I+Z_{0} I\right] \subset[I, I]+Z_{0}[I, I] \subset I$. Hence $I=[K, K]$.

We note that the set $S$ is a vector space over $Z_{0}$ and has a basis $\left\{u_{i} \mid i=1, \cdots, 8\right\}$ such that $u_{i}^{2} \in Z_{0}$ and $q\left(u_{i}, u_{j}\right)=0$ if $i \neq j$. The $u_{i}$ 's will also be a basis for $\mathscr{C}$ over $Z$.

REMARK. $K^{-}$is necessarily Malcev since it is a subalgebra of the Malcev algebra $\mathscr{C}^{-}$. When the involution is standard, $K^{-}$is a simple non-Lie-Malcev algebra; when it is nonstandard, $K^{-}$is a simple Lie algebra (Sagle [12]).

LEMmA 4. If $R$ is a *-simple alternative ring with identity element and $1 / 2 \in R$, then $S^{+}$is a simple Jordan ring.

Proof. A *-simple ring is either simple or the direct sum of a simple ring $B$ and its opposite, $B^{o p}$, under the exchange involution.

If $R$ is simple, it is either associative and $S^{+}$is simple by Herstein [3], or it is a Cayley-Dickson algebra and is simple by Lemmas 2 and 3 . 
Suppose now $R=B \oplus B^{o p}$ under the exchange involution. Then $S^{+}$is isomorphic to $B^{+}$. If $B$ is associative, $B^{+}$is simple by Herstein [3]. If $B$ is a Cayley-Dickson algebra $B^{+}$is simple since the norm form is nondegenerate on $B$.

3. When symmetric elements are invertible or nilpotent. An alternative division ring is either an associative division ring or a Cayley-Dickson division algebra. Obviously any nonzero symmetric element is an alternative division ring with involution is invertible. More can be said.

THEOREM 1. Let $R$ a *-simple alternative ring with identity element $e$ and $1 / 2 \in R$. Each nonzero symmetric element is invertible if and only if $R$ is one of

(i) an alternative division ring,

(ii) the direct sum of an alternative division ring and its opposite, with the involution interchanging the summands,

(iii) the ring of $2 \times 2$ matrices over a field with the symplectic involution, or

(iv) a split Cayley-Dickson algebra with the standard involution.

Proof. If $R$ is associative, then each nonzero symmetric element is invertible in the nucleus and Jacobson [6], Theorem 8, p. 170, applies.

If $R$ is not associative and is *-simple it is either a CayleyDickson algebra or the direct sum of a Cayley-Dickson algebra with its opposite under the exchange involution. If $R$ is a division algebra or the direct sum of a division algebra and its opposite under the exchange involution, clearly any nonzero symmetric element is invertible. If $R$ is a split Cayley-Dickson algebra, the involution must be the standard involution.

To see this note that if $\mathscr{C}$ is split and $Z \cong S$, then by Lemma $2, S$ is a five dimensional subspace of $\mathscr{C}$ over $Z$. Since $\mathscr{C}$ is split, a nonisotropic subspace has dimension at most four by Witt's theorem, so $S$ cannot be nonisotropic, hence there must be some $0 \neq s \in S$ such that $q(s, s)=0$. That is, $S$ must have a noninvertible element. Hence, if $*$ is of the first kind, it is the standard involution.

For an involution of the second kind, we use a different approach. Since $\mathscr{C}$ is split, it has a pair of orthogonal idempotents, $e_{1}$ and $e_{2}$, where $e_{1}+e_{2}=e$ and the subspace $\mathscr{C}_{12}=\left\{x_{12} \mid e_{1} x_{12}=x_{12}, x_{12} e_{2}=x_{12}\right\}$ is nonzero. If $x \in \mathscr{C}_{12}$, then $x^{2}=0$. Let $x$ be a nonzero element of $\mathscr{C}_{12}$. If $x x^{*}$ were invertible, then $x$ would have a right inverse, $z$. Then $x^{2}=0$ implies $x(x z)=x e=x=0$, a contradiction since $x \neq 0$. Likewise $x^{*} x=0, x+x^{*}=0$, and then, $x^{*}=-x$. If $0 \neq \lambda \in Z$ and $\lambda^{*}=-\lambda$, 
then $\lambda x$ would be symmetric, nonzero and non-invertible. So $*$ is not of the second kind.

Since the direct sum of a split Cayley-Dickson algebra and its opposite under the exchange involution would have symmetric divisors of zero, we rule this possibility out.

THEOREM 2. Let $R$ be an alternative ring with identity element $e$ and $1 / 2 \in R$ with involution *. Then every symmetric element of $R$ is nilpotent or invertible if and only if the following two conditions both hold:

(a) $\operatorname{Rad} R$ is a *-ideal in which every symmetric element is nilpotent.

(b) The quotient ring $R / \operatorname{Rad} R$ is isomorphic to one of the four possibilities of Theorem 1.

Proof. $\operatorname{Rad} R$ is invariant under all automorphisms and antiautomorphisms, hence $\operatorname{Rad} R$ is a $*$-ideal. Since there are no invertible elements in $\operatorname{Rad} R$, the symmetric elements in $\operatorname{Rad} R$ must all be nilpotent and condition (a) holds.

Let $P$ be the set of symmetric elements of the center of $R / \operatorname{Rad} R$. Then $P$ is a subring which contains no nilpotent elements since $R / \operatorname{Rad} R$ contains no nonzero nilpotent ideals. Hence the nonzero elements of $P$ are invertible, and so $P$ is a field since the inverse of an element of $P$ is clearly in $P$ again. Since $1 / 2 \in R, 1 / 2$ remains in $R / \operatorname{Rad} R$. Since the nonzero homomorphic image of a nilpotent (invertible) element is nilpotent (invertible) it is clear that $R / \operatorname{Rad} R$ satisfies the same hypothesis as $R$. Thus it suffices to establish condition (b) under the added hypothesis that $R$ is radical free.

Suppose now that $B$ is a proper $*$-ideal of $R$. Since $B$ can contain no invertible elements and still be proper, the symmetric elements of $B$ are all nilpotent. By Lemma $1, B$ is a radical $\operatorname{ring}, B \subseteq \operatorname{Rad} R$; but this last is zero.

Thus $R$ is *-simple. By Lemma $4, S^{+}$is a simple Jordan algebra with identity whose elements are either nilpotent or invertible. By simplicity, $S^{+}$contains no nonzero nil ideals, so we may conclude by Osborn [11] that $S^{+}$contains no nonzero nilpotent elements. Hence $R$ is a *-simple alternative ring with identity such that each nonzero symmetric element is invertible. The result now follows from Theorem 1.

Conversely, let $R$ be an alternative ring with involution and with identity satisfying conditions (a) and (b). We wish to show that every symmetric element $s$ of $R$ is either nilpotent or invertible. Since $s \in S, \bar{s}$ is a symmetric element of $R / \operatorname{Rad} R$ under the induced involution. If $\bar{s}=0$, then $s \in \operatorname{Rad} R$ and is nilpotent by (a). If 
$\bar{s} \neq 0$, then $\bar{s}$ is invertible in $R / \operatorname{Rad} R$ by (b). But $s$ is invertible in $R$ if and only if $\bar{s}$ is invertible in $R / N$ for any quasi-invertible ideal $N$.

As in Osborn [11], we get

THEOREM 3. If $R$ is an alternative algebra with involution over an uncountable field such that every symmetric element is nilpotent, then $R$ is a nil-algebra.

4. Radical free alternative rings. In this section $R$ is a radical free ring with identity $e$ and $1 / 2 \in R$ and with involution $*$ such that each symmetric element $s$ is either nilpotent or some (right) multiple of $s$ is a symmetric idempotent. Furthermore, $R$ has a set $\left\{e_{1}\right\}_{i=1}^{n}$ of pairwise orthogonal, symmetric idempotents whose sum is one and if $\left\{f_{i}\right\}_{i=1}^{m}$ is any such set of idempotents whose sum is one, then $m \leqq n$.

Let $R=\sum R_{i j},(i, j=1,2, \cdots, n)$, be the Pierce decomposition of $R$ relative to the set $\left\{e_{i}\right\}_{i=1}^{n}$ of pairwise orthogonal symmetric idempotents.

Lemma 5. $R_{i i},(i=1,2, \cdots, n)$, is isomorphic to one of the four possibilities of Theorem 1 .

Proof. $R_{i i},(i=1,2, \cdots, n)$, is radical free since $R$ is (McCrimmon [9]). $\quad R_{i i}$ is a $*$-invariant subring of $R$. Each symmetric element $s$ of $R_{i i}$ is either nilpotent or there is some $p \in R$ such that $s p$ is a nonzero symmetric idempotent. Since $R_{i i}$ is radical free, not all $s \in R_{i i}$ are nilpotent, so there are some $p \in R$ and $s \in R_{i i}$ with $s p=$ $e=e^{*}$ nonzero symmetric idempotent. Furthermore $s p \in R_{i i}$ since $s p=e=e^{*}$ and $e=e e^{*}=(s p)\left(p^{*} s\right)=s\left(p p^{*}\right) s \in R s \subset R_{i i}$ by the Moufang identities. If $e=s p$ is not $e_{i}$, we would have

$$
e=\sum_{\substack{j=1 \\ j \neq i}}^{n} e_{j}+\left(e_{i}-s p\right)+s p
$$

and $R$ would have $n+1$ pairwise orthogonal symmetric idempotients. Hence $s p=e_{i}$, the identity of the ring $R_{i i}$. That is each symmetric element of $R_{i i}$ is either nilpotent or invertible and Theorem 2 applies.

LEMMA 6. Each nonzero ideal of $R$ contains a minimal (nonzero) ideal and has nonzero intersection with some $R_{i i}$.

Proof. Any ideal $I$ of $R$ such that $I \subseteq \sum_{i \neq j} R_{i j}$ is the zero ideal. Let $x$ be a nonzero element of an ideal contained in $\sum_{i \neq j} R_{i j}$. Then 
$x=\sum x_{i j}$ as in Zorn [17] and [18]. $x^{k}$ is a sum of products $x_{i_{1} i_{2}} x_{i_{2} i_{3}} x_{i_{3} i_{4}} \cdots x_{i_{k} i_{k+1}}$ (parenthesis unnecessary - the $x_{i j}$ generate an associative subalgebra since in an extension it is contained in the subalgebra generated by the two elements $x$ and $y=\sum \alpha_{i} e_{i}$ for distinct, nonzero $\left.\alpha_{i}\right)$ where no $i_{a}=i_{b}(1 \leqq a \leqq b \leqq k+1)$ or else $x_{i_{a} i_{a+1}} x_{i_{a+1} i_{a+2}} \cdots x_{i_{b-1} i b}$ would be in $I \cap R_{i_{a} i_{b}}=I \cap R_{i_{a} i_{a}}=0$ and the product is zero. Thus all $i_{1}, \cdots, i_{k+1}$ are distinct and $k+1 \leqq n, k \leqq$ $n, x^{n}=0$. Hence $I$ would be a nil-ideal. But $R$ is radical free and hence $I=0$.

Let $I$ be a nonzero ideal in $R$ and let $\mathscr{J}$ be the family of all nonzero ideals of $R$ contained in $I$. We claim that if $A$ is a nest in $\mathscr{F}$, then $\bigcap_{a \in A} a \neq 0$.

For each $i, i=1,2, \cdots, n$, the family $\left\{a \cap R_{i i} \mid a \in A\right\}$ is a nest of ideals in the ring $R_{i i}$. By Lemma 5 , each of the rings $R_{i i}$ has d.c.c. for right ideals; that is, there is some $a_{i} \in A$ such that $a_{i} \cap R_{i i}$ is a minimal ideal in $R_{i i}$. Pick $a_{0}$ minimal in the set $\left\{a_{i}\right\}_{i=1}^{n}, a_{0} \cap R_{i i}$ cannot be zero for all $i$, otherwise $a_{0}$ would be zero. Hence $\bigcup_{i=1}^{n}\left(a_{0} \cap R_{i i}\right) \neq$ 0 and is contained in each $a \in A$, that is, $\bigcap_{a \in A} a \neq 0$.

The minimal principal applies and the ideal $I$ contains a minimal, nonzero ideal.

LEMma 7. $R$ does not contain an infinite descending chain of direct summands.

Proof. Let $\left\{I_{i}\right\}_{i=1}^{n_{0}}$ be a set of ideals of $R$ such that $I_{j} \cap I_{i}=0$ if and only if $i \neq j$. Then $n_{0} \leqq 2 n$.

Any ideal of $R$ intersects $R_{i i}, i=1,2, \cdots, n$, in an ideal. By Lemma 5, each $R_{i i}$ has at most two nonzero ideals $L$ and $K$ such that $L \cap K=0$. Then at most two of the ideals $\left\{I_{i}\right\}_{i=1}^{n_{0}}$ can intersect $R_{i i}$ nontrivially. Since each $I_{k}$ must intersect some $R_{i i}$ nontrivially by Lemma 6 , there can be at most $2 n$ ideals in $\left\{I_{n}\right\}_{i=1}^{n_{0}}$.

Let $R=R_{0} \supset R_{1} \supset R_{2} \supset \cdots \supset R_{m}$ be a finite descending chain of direct summands of $R$. Thus $R=R_{i} \oplus R_{i}^{\prime}$ for each $i=1,2, \cdots, m$. By the modular law for ideals,

$$
R_{i}=R_{i+1} \oplus\left(R_{i} \cap R_{i+1}^{\prime}\right) .
$$

Then $R$ is a direct sum of $m+1$ ideals:

$$
R=R_{m} \oplus \sum_{i=1}^{m}\left(R_{m-i} \cap R_{m+1-i}^{\prime}\right) .
$$

That is, $R$ has a set $S$ of $m+1$ nonzero ideals $\left\{I_{i}\right\}_{i=1}^{m+1}$ such that $I_{i} \cap I_{j}=0$ if $I_{i} \neq I_{j}$. Hence $m+1 \leqq 2 n$.

COROLLARY 1. $R$ has d.c.c. on right ideals. 
Proof. Since $R$ is semiprime, does not contain an infinite descending chain of direct summands, and every ideal contains a minimal ideal, the hypothesis of Slater [15], Theorem C, is met and

$$
R=\mathscr{C}_{1} \oplus \mathscr{C}_{2} \oplus \cdots \oplus \mathscr{C}_{m} \oplus \mathscr{A}
$$

where each $\mathscr{C}_{i}$ is a Cayley-Dickson algebra and $\mathscr{A}$, the maximal nuclear ideal of $R$, is associative. $\mathscr{A}$ is a $*$-ideal, has an identity, $1 / 2 \in \mathscr{A}$, and by Wene [16], $\mathscr{A}$ has d.c.c. on right ideals.

\section{Main result.}

THEOREM 4. If $R$ is an alternative ring with unit element and $1 / 2 \in R$ having an involution such that each symmetric element $s$ is either nilpotent or some right multiple of $s$ is a nonzero symmetric idempotent and there is a positive integer $n$ such that each set of pairwise orthogonal symmetric idempotents summing to the identity has $n$ or less elements, then $\operatorname{Rad} R$ is a *-ideal in which every symmetric element is nilpotent and $R / \operatorname{rad} R$ can be expressed, uniquely up to order, as a direct sum of *-simple rings $R_{1} \oplus \cdots \oplus$ $R_{m}$ where each $R_{i}$ is either

(a) $\mathscr{C} \oplus \mathscr{C}^{\circ p}$ for a Cayley-Dickson algebra under the exchange involution,

(b) a Cayley-Dickson algebra $\mathscr{C}$ with any involution,

(c) $A \oplus A^{o p}$ for a simple artinian associative ring $A$ with the exchange involution,

(d) a simple artinian associative ring with involution.

Proof. Since $\operatorname{Rad} R$ cannot contain nonzero idempotents any $s \in$ $S \cap \operatorname{Rad} R$ is nilpotent.

In $R / \operatorname{Rad} R$ any element $\bar{s}$ that is symmetric under the induced involution is either nilpotent or some right multiple of $\bar{s}$ is a nonzero idempotent. If $\left\{e_{i}\right\}_{i=1}^{n}$ is a set of pairwise orthogonal symmetric idempotents where sum is the identity of $R$, then $\left\{\bar{e}_{i}\right\}_{i=1}^{n}$ is a set of pairwise orthogonal symmetric idempotents in $R / \operatorname{Rad} R$ whose sum is the identity. On the other hand, any set of pairwise orthogonal symmetric idempotents in $R / \operatorname{Rad} R$ can be lifted to a set of pairwise orthogonal symmetric idempotents of $R$. The Jordan ring

$$
\frac{S^{+}+\operatorname{Rad} R^{+}}{\operatorname{Rad} R^{+}} \text {is isomorphic to the Jordan ring } \frac{S^{+}}{S^{+} \cap \operatorname{Rad} R^{+}}
$$

and by Jacobson [7] p. 149, idempotents can be lifted since $S^{+} \cap$ $\operatorname{Rad} R^{+}$is nil.

Thus $R / \operatorname{Rad}=\widetilde{R}$ is radical free and has a set of $n$ pairwise orthogonal symmetric idempotents whose sum is one and any such 
set of pairwise orthogonal symmetric idempotents has $n$ or less idempotents. By Corollary 1, $\widetilde{R}$ has d.c.c. on right ideals. But then $\widetilde{R}$ is a direct sum of ${ }^{*}$-simple rings each with d.c.c. on right ideals. $\widetilde{R}$ is a direct sum of simple rings. Suppose $\widetilde{R}=R_{1} \oplus R_{2}$, where $R_{1}$ and $R_{2}$ are simple rings. Then $\widetilde{R}=R_{1}^{*} \oplus R_{2}^{*}$ and $R_{1}=\left(R_{1} \cap R_{1}^{*}\right) \oplus$ $\left(R_{1} \cap R_{2}^{*}\right)$. If $R_{\mathrm{1}} \cap R_{1}^{*}=0, R_{1}^{*} \subset R_{2}$ and $R=R_{1} \oplus R_{1}^{*}=R_{1} \oplus R_{1}^{o p}$. On the other hand, if $R_{1} \cap R_{1}^{*} \neq 0$ then $R_{2} \cap R_{1}^{*}=0$. Otherwise $R_{1}^{*}=$ $\left(R_{1}^{*} \cap R_{1}\right) \oplus\left(R_{1}^{*} \cap R_{2}\right)$. Thus we have $R_{1}=R_{1} \oplus R_{2}$ and $R_{1}^{*}=R_{1}$ and $R_{2}^{*}=R_{2}$. An induction argument on the number of direct summands of $R$ now gives the desired result.

The author wishes to thank Professor Robert H. Oehmke for the suggestion of the problem and for his helpful criticism. Part of these results are contained in the author's doctorial dissertation at the University of Iowa.

\section{REFERENCES}

1. H. Braun and M. Koecher, Jordan-Algebren, Springer-Verlag, Berlin, Germany, 1966.

2. L. I. Golovena, Commutative radical rings, Izestiya Akad. Nauk. SSSR., Ser. Mat., 14 (1950), 449-472.

3. I. N. Herstein, Topics in Ring Theory, The University of Chicago Press, Chicago, Illinois, 1969.

4. — Rings with Involution, The University of Chicago Press, Chicago, Illinois, 1976.

5. N. Jacobson, Composition algebras and their automorphisms, Cir. Math. Palermo, Rendicoti, 7 (2) 1958, 55-80.

6. - Structures and Representations of Jordan Algebras, Amer. Math. Soc., Colloquium Publications XXXIX, Providence, Rhode Island, 1968.

7. N. Jacobson and C. E. Rickart, Homomorphisms of Jordan rings of self-adjoint elements, Trans. Amer. Math. Soc., 72 (1952), 310-322.

8. E. Kleinfeld, Simple alternative rings, Ann. of Math., 58 (1953), 544-547.

9. K. McCrimmon, A characterization of the Jacobson-Smiley radical, J. Algebra, 18 (1971), 565-573.

10. J. M. Osborn, Jordan algebras of capacity two, Proc. Nat. Acad. Sci. USA, 57 (1967), 582-589.

11. Jordan and associative rings with nilpotent and invertible elements, J. Algebra, 15 (1970), 301-308.

12. A. A. Sagle, Malcev algebras, Trans. Amer. Math. Soc., 101 (1961), 426-458.

13. - On anti-commutative algebras with an invariant form, Canad. J. Math., 16 (1964), 370-378.

14. R. D. Schafer, Introduction to Nonassociative Algebras, Academic Press, New York, 1966.

15. M. Slater, Alternative rings with d.c.c., I, J. Algebra, 11 (1969), 102-110.

16. G. P. Wene, On a result of Osborn, Proc. Amer. Math. Soc., 69 (1978), 11-15.

17. M. Zorn, Theorie der alternativen Ringe, Abh. Math. Sem. Univ. Hamburg, 8 (1930), 123-147.

18. - Alternative rings and related questions $I$ : Existence of the radical, Ann. of Math., 42 (1941), 676-686.

Received April 20, 1978 and in revised form August 14, 1979. 


\section{PACIFIC JOURNAL OF MATHEMATICS}

\section{EDITORS}

DONALD BABBITT (Managing Editor)

University of California

Los Angeles, CA 90024

Hugo RossI

University of Utah

Salt Lake City, UT 84112

C. C. MOORE and ANDREW OGG

University of California

Berkeley, CA 94720

\section{J. DugunduI}

Department of Mathematics

University of Southern California

Los Angeles, CA 90007

R. Finn and J. Milgram

Stanford University

Stanford, CA 94305

\section{ASSOCIATE EDITORS}
E. F. BeCKenBACH
B. H. NeumanN
F. WOLF
K. YosHIDA

\section{SUPPORTING INSTITUTIONS}

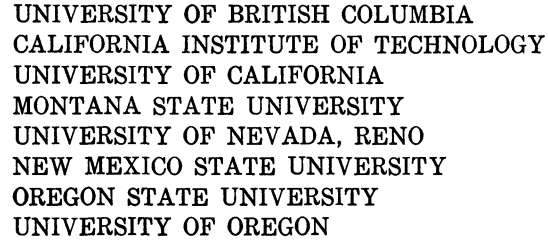

UNIVERSITY OF BRITISH COLUMBIA CALIFORNIA INSTITUTE OF TECHNOLOGY UNIVERSITY OF CALIFORNIA

MONTANA STATE UNIVERSITY

UNIVERSITY OF NEVADA, RENO

NEW MEXICO STATE UNIVERSITY

OREGON STATE UNIVERSITY UNIVERSITY OF OREGON

\author{
UNIVERSITY OF SOUTHERN CALIFORNIA \\ STANFORD UNIVERSITY \\ UNIVERSITY OF HAWAII \\ UNIVERSITY OF TOKYO \\ UNIVERSITY OF UTAH \\ WASHINGTON STATE UNIVERSITY \\ UNIVERSITY OF WASHINGTON
}

The Supporting Institutions listed above contribute to the cost of publication of this Journal, but they are not owners or publishers and have no responsibility for its content or policies.

Mathematical papers intended for publication in the Pacific Journal of Mathematics should be in typed form or offset-reproduced, (not dittoed), double spaced with large margins. Please do not use built up fractions in the text of the manuscript. However, you may use them in the displayed equations. Underline Greek letters in red, German in green, and script in blue. The first paragraph or two must be capable of being used separately as a synopsis of the entire paper. Please propose a heading for the odd numbered pages of less than 35 characters. Manuscripts, in triplicate, may be sent to any one of the editors. Please classify according to the scheme of Math. Reviews, Index to Vol. 39. Supply name and address of author to whom proofs should be sent. All other communications should be addressed to the managing editor, or Elaine Barth, University of California, Los Angeles, California, 90024.

50 reprints to each author are provided free for each article, only if page charges have been substantially paid. Additional copies may be obtained at cost in multiples of 50 .

The Pacific Journal of Mathematics is issued monthly as of January 1966. Regular subscription rate: $\$ 84.00$ a year (6 Vols., 12 issues). Special rato: $\$ 42.00$ a year to individual members of supporting institutions.

Subscriptions, orders for numbers issued in the last three calendar years, and changes of address shoud be sent to Pacific Journal of Mathematics, P.O. Box 969, Carmel Valley, CA 93924, U.S.A Old back numbers obtainable from Kraus Periodicals Co., Route 100, Millwood, NY 10546.

PUBLISHED BY PACIFIC JOURNAL OF MATHEMATICS, A NON-PROFIT CORPORATION

Printed at Kokusai Bunken Insatsusha (International Academic Printing Co., Ltd.). 8-8, 3-chome, Takadanobaba, Shinjuku-ku, Tokyo 160, Japan.

Copyright (C) 1980 by Pacific Jounal of Mathematics Manufactured and first issued in Japan 


\section{Pacific Journal of Mathematics \\ Vol. 90, No. $2 \quad$ October, 1980}

Frank Hayne Beatrous, Jr., Hölder estimates for the $\bar{\partial}$ equation with a support condition ..................................... 249

Charles L. Belna, Michael Jon Evans and Paul Humke, Planar continua

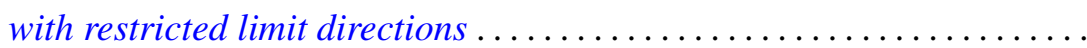

Leon Brown and Takashi Ito, Classes of Banach spaces with unique isometric preduals................................. 261

V. K. Deshpande, Completions of Noetherian hereditary prime rings ..... . 285

Deepak Dhar, Asymptotic enumeration of partially ordered sets . . . . . . . 299

Zeev Ditzian, On interpolation of $L_{p}[a, b]$ and weighted Sobolev

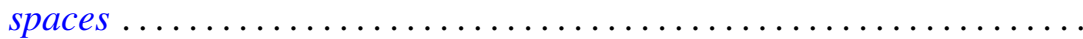

Andrew George Earnest, Congruence conditions on integers represented by

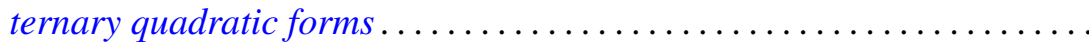

Melvin Faierman, Bounds for the eigenfunctions of a two-parameter system of ordinary differential equations of the second order ..............

Hector O. Fattorini, Vector-valued distributions having a smooth

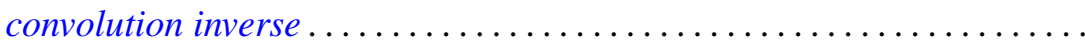

Howard D. Fegan, The spectrum of the Laplacian on forms over a Lie

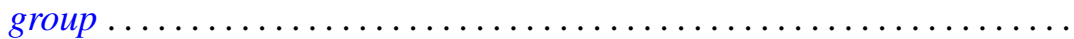

Gerald Leonard Gordon, On the degeneracy of a spectral sequence associated to normal crossings..

S. Madhavan, On bisimple weakly inverse semigroups ... 397

Françoise Mathot, On the decomposition of states of some

Roger McCann, Embedding asymptotically stable dynamical systems into radial flows in $l_{2}$

Michael L. Mihalik, Ends of fundamental groups in shape and proper homotopy...............................

Samuel Murray Rankin, III, Boundary value problems for partial functional differential equations .

Randy Tuler, Arithmetic sums that determine linear characters on $\Gamma(N)$

Jeffrey D. Vaaler, On linear forms and Diophantine approximation ..

G. P. Wene, Alternative rings whose symmetric elements are nilpotent or a right multiple is a symmetric idempotent. . 